\title{
The effect of the dynamic splitting of the conductivity state in homogeneous electric field
}

\author{
A.D. Suprun, L.V. Shmeleva \\ Faculty of Physics, Kyiv National T. Shevchenko University, \\ Glushkova Ave., 4, Kyiv, Ukraine
}

Received April 11, 2017

The features of a completely homogeneous external electrostatic field effect on the properties of a quasiparticle of the electron type injected into the conduction band of a semiconductor or dielectric are considered. As applied to this case (completely homogeneous external field), the equations obtained earlier are analyzed, which provide a consistent quantum (basic) and classical (in terms of quasiparticles) descriptions. It is shown that these equations reflect regularities that do not allow the "direct" application of the external field both in the basic (quantum) and in the effective (in terms of quasiparticles) descriptions. These regularities and the corresponding equations are one of the possible causes for the experimentally observed anomalous splitting of the conductivity state in strong fields. In the article this splitting was called a dynamic splitting. A satisfactory agreement with the experiment was obtained.

Keywords: Quasiparticle, electrostatic field, anomalous splitting, conduction band, electric current.

Рассмотрены особенности влияния полностью однородного внешнего әлектростатического поля на свойства квазичастицы типа электрона, инжектированного в зону проводимости полупроводника или диэлектрика. Применительно к этому случаю (полностью однородного внешнего поля) проанализированы полученные ранее уравнения, которые обеспечивают согласованное квантовое (базовое) и классическое (в терминах квазичастиц) описания. Показано, что эти уравнения отражают закономерности, не допускающие "прямое" применение внешнего поля как в базовом (квантовом), так и в әффективном (в терминах квазичастиц) описаниях. Эти закономерности и соответствующие им уравнения являются одной из возможных причин экспериментально наблюдаемого аномального расщепления состояния проводимости у сильных полях. Получено удовлетворительное согласие с экспериментом.

Ефект динамічного розщеплення стану провідності в однорідному електричному полі. А.Д. Супрун, Л.В. Шилельова

Розглянуто особливості впливу повністю однорідного зовнішнього електростатичного поля на властивості квазічастинки типу електрона, інжектованого в зону провідності напівпровідника або діелектрика. Стосовно до цього випадку (повністю однорідного зовнішнього поля) проаналізовано отримані раніше рівняння, які забезпечують узгоджене квантове (базове) і класичне (у термінах квазічастинок) описи. Показано, що ці рівняння відображають закономірності, що не допускають "пряме" застосування зовнішнього поля як у базовому (квантовому), так і в ефективному (у термінах квазічастинок) описах. Ці закономірності й відповідні їм рівняння $є$ однією з можливих причин експериментально спостережуваного аномального розщеплення стану провідності в сильних полях. Отримано задовільне узгодження з експериментом. 


\section{Introduction}

In article [1], the features of the dynamics of a free electron in the conduction band of a crystal were analyzed. It was shown that they are based on such a basic characteristic of excited media, as the energy or frequency dispersion from the wave vector [2 - 7]. Later in [8], a general analysis of the effect of an external electrostatic field of weakly variable spatial configuration on the features of free electron dynamics in the conduction band was made. In this case, special attention, as before [1], was paid to the fact that the quantum (basic) and classical descriptions were in compliance. In [9] some important applications of the results were analyzed.

Here we present the case of an electron in a completely homogeneous electrostatic field. It requires an individual consideration. It is shown that this case, at first glance, should not have feat ures, but they are exist. Similar features are observed during experiment [10]. As it is shown in the article, they occur due to the fact that the "behaviour" of an electron in the conduction band as a quantum object and as a classical object must be coordinated. This, in turn, does not allow for the "direct"application of the external field for describing electron dynamics in both descriptions (both quantum and classical) and is one of the possible causes of the experimentally observed anomalous splitting of the conductivity state in strong fields. A satisfactory compliance with the observed splitting was obtained in experiments for PNB polymers. If the considered splitting mechanism is unique, then on the basis of the results obtained, the width of the conduction band for these polymers can be evaluated, which in this case is $0.8 \mathrm{eV}$. It is also shown that in one of the states obtained, the electron velocity is practically half than in the other. On the basis of this, the possibility of the existence of an anomalous current behaviour is shown.

\section{Materials and methods}

\subsection{General conditions for consistent quantum and classical descriptions of an electron in a solid in an external field}

These conditions for the case of a weakly variable spatial configuration of the field have the form of four relations, discussed in detail in [8,9]. The analysis of an electron dynamics (as a quasiparticle) injected into the conduction band is based on the equations of motion:

$$
\dot{\mathbf{r}_{0}}=\boldsymbol{\beta}(\mathbf{p}), \quad \dot{\mathbf{p}}=\mathbf{G} .
$$

Here $\boldsymbol{\beta}(\mathbf{p})$ is the dimensionless velocity of a quasiparticle with components $\beta\left(p_{\alpha}\right): \boldsymbol{\beta}(\mathbf{p})=\mathbf{e}_{\alpha} \beta\left(p_{\alpha}\right)$, where $\beta\left(p_{\alpha}\right) \equiv \sin \left(p_{\alpha}\right)$. Components of a dimensionless momentum $\mathbf{p}$ are marked with $p_{\alpha} . \mathbf{G}$ is dimensionless representation of the force produced by an external field in a "global"frame of reference (associated with a crystal lattice). Further consideration of the "electron in a solid - external field"system as conservative and potential, we confine to the fact that this force depends only on $\mathbf{r}_{0}$. In this sense, the variables $\mathbf{p}$ and $\mathbf{r}_{0}$ are canonically conjugated.

In $[8,9]$ it was shown that these equations have exactly the form (1) only under the conditions

$$
\begin{gathered}
\left(\widehat{\mu}^{-1} \mathbf{F} \cdot \frac{\partial \boldsymbol{\xi}}{\partial p_{\alpha}}\right)+\left(\boldsymbol{\Phi} \cdot \frac{\partial \mathbf{r}_{*}}{\partial p_{\alpha}}\right)+ \\
+\left(\left(\boldsymbol{\xi}-\mathbf{r}_{0}\right) \cdot \frac{\partial}{\partial p_{\alpha}}\left(\widehat{\mu}^{-1} \mathbf{F}\right)\right)=0 ; \\
(\mathbf{p} \cdot \dot{\boldsymbol{\xi}})+\left(\left\{\boldsymbol{\xi}-\mathbf{r}_{0}\right\} \cdot \mathbf{G}\right)=0 ; \\
\widehat{\mu}^{-1} \mathbf{F}-\mathbf{G}=\boldsymbol{\sigma} ; \\
\widehat{\mu}^{-1} \mathbf{F}+\mathbf{G}=\mathbf{\Pi}_{\mathbf{r}_{*},}
\end{gathered}
$$

where $\sigma$ is a vector with components: 


$$
\begin{aligned}
& \sigma_{\alpha} \equiv\left(\widehat{\mu}^{-1} \mathbf{F} \cdot \frac{\partial \boldsymbol{\xi}}{\partial x_{0}^{\alpha}}\right)+\left(\boldsymbol{\Phi} \cdot \frac{\partial \mathbf{r}_{*}}{\partial x_{0}^{\alpha}}\right)+ \\
& +\left(\left(\boldsymbol{\xi}-\mathbf{r}_{0}\right) \cdot \frac{\partial}{\partial x_{0}^{\alpha}}\left(\widehat{\mu}^{-1} \mathbf{F}\right)\right)
\end{aligned}
$$

were $\alpha=1,2,3$ and $\dot{\xi}$, as usual, represent the total time derivative. It was shown that these conditions are essentially the conditions for the dynamics of the quasiparticle to be Hamiltonian. On the one hand, they ensure the consistency of the general consideration with the case of a free quasiparticle $(\mathbf{G}=0)$, and on the other hand, the consistency of the quantum and classical descriptions.

Equations (2) - (5) are formulated with respect to four unknown vector quantities: $\mathbf{G}, \mathbf{F}, \boldsymbol{\xi}$ and $\mathbf{r}_{*}$. Two of these vectors are dimensionless forces $\mathbf{G}$ and $\mathbf{F}$. The force $\mathbf{G}$, as it was already noted, is a representation of the force created by the external field in the "global"frame of reference (associated with the crystal lattice). And the force $\mathbf{F}$ is a dimensionless representation of the same force, but in the "local"frame of reference, associated with the point $\mathbf{r}_{0}$ of conditional localization of a quasiparticle (an electron in the conduction band).

The other two unknown vectors are $\boldsymbol{\xi}$ and $\mathbf{r}_{*}$. Vector $\boldsymbol{\xi}$ and its components $\xi_{1}, \xi_{2}, \xi_{3}$ have a meaning of dimensionless length, to which this feature of the considered system is transferred, as "local" (in the frame of reference connected with the point $\mathbf{r}_{0}$ ) eigenvalue in accordance with definition:

$$
\varepsilon=\left(\boldsymbol{\xi} \cdot \widehat{\mu}^{-1} \mathbf{F}\right)
$$

where $\varepsilon$ is a "local"eigenvalue. And $\widehat{\mu}$ hereinafter- is the dimensionless diagonal tensor of the dynamic effective mass with components:

$$
\mu_{\alpha} \equiv 1 / \cos \left(p_{\alpha}\right)
$$

Vector $\mathbf{r}_{*}$ is a dimensionless representation of the point of conditional localization of the source of the field in a linear approximation in quantum space variables. And, finally, the vector $\boldsymbol{\Phi}$ is determined through its components as follows:

$$
\Phi_{\alpha} \equiv\left(\mathbf{r}_{*} \cdot \frac{\partial \boldsymbol{\Pi}_{\mathbf{r}_{*}}}{\partial x_{*}^{\alpha}}\right),
$$

where $\boldsymbol{\Pi}_{\mathbf{r}_{*}}$ is a dimensionless representation of a given external force, which, in general, depends on the variable $\mathbf{r}_{*}$ with components $x_{*}^{\alpha}$.

Conditions (2) - (4) (condition (4) with taking into account of the definition (6)), have the form of differential equations for determining the vector functions $\mathbf{F}, \boldsymbol{\xi}$ and $\mathbf{r}_{*}$. Equation (5) actually determines the force $\mathbf{G}: \mathbf{G}=\mathbf{\Pi}_{\mathbf{r}_{*}}-\widehat{\mu}^{-1} \mathbf{F}$.

\subsection{Reduction of the basic relations for the case of a completely spatial homogeneous external field}

The term "completely spatial homogeneous external field" is usually meant a situation where a given force, determined by the relation $\boldsymbol{\Pi}_{\mathbf{r}_{*}}=\nabla\left(v_{\mathbf{r}_{*}}\right)$, is reduced to the condition $\boldsymbol{\Pi}_{\mathbf{r}_{*}}=\boldsymbol{\Pi}=$ const. In this case it is obvious that the dimensionless potential energy: $v_{\mathbf{r}_{*}}=\left(\mathbf{r}_{*} \cdot \boldsymbol{\Pi}\right)$, and from the definition (9) it follows that $\Phi=0$. Then condition (2) and definition (6) are simplified to the form:

$$
\begin{gathered}
\nabla_{\mathbf{p}}\left(\left\{\boldsymbol{\xi}-\mathbf{r}_{0}\right\} \cdot \widehat{\mu}^{-1} \mathbf{F}\right)=\mathbf{0} \\
\sigma=\widehat{\mu}^{-1} \mathbf{F}+\nabla_{\mathbf{r}_{0}}\left(\left\{\boldsymbol{\xi}-\mathbf{r}_{0}\right\} \cdot \widehat{\mu}^{-1} \mathbf{F}\right),
\end{gathered}
$$

and the whole system (2) - (5) takes the form:

$$
\nabla_{\mathbf{p}}\left(\left\{\xi-\mathbf{r}_{0}\right\} \cdot \widehat{\mu}^{-1} \mathbf{F}\right)=0
$$




$$
\begin{gathered}
(\mathbf{p} \cdot \dot{\boldsymbol{\xi}})+\left(\left\{\boldsymbol{\xi}-\mathbf{r}_{0}\right\} \cdot \mathbf{G}\right)=0 ; \\
\mathbf{G}=-\nabla_{\mathbf{r}_{0}}\left(\left\{\boldsymbol{\xi}-\mathbf{r}_{0}\right\} \cdot \widehat{\mu}^{-1} \mathbf{F}\right) \\
\mathbf{G}=\mathbf{\Pi}-\widehat{\mu}^{-1} \mathbf{F},
\end{gathered}
$$

and now defines the vectors $\mathbf{F}, \boldsymbol{\xi}, \mathbf{G}$ (vector $\mathbf{r}_{*}$ "fall out" from consideration due to constraint $\boldsymbol{\Pi}_{\mathbf{r}_{*}}=\mathbf{\Pi}=$ const). As $\mathbf{p}$ and $\mathbf{r}_{0}$ are considered as independent Hamiltonian variables (canonically conjugate pair), then equation (10) has a general solution (the integral of motion): $\left(\left\{\boldsymbol{\xi}-\mathbf{r}_{0}\right\} \cdot \hat{\mu}^{-1} \mathbf{F}\right)=U\left(\mathbf{r}_{0}\right)$, where $U\left(\mathbf{r}_{0}\right)$ - an arbitrary function that has the meaning of potential energy. Equation (12), in this case, takes the form: $\mathbf{G}=-\nabla_{\mathbf{r}_{0}}\left(U\left(\mathbf{r}_{0}\right)\right)$, thereby transferring the uncertainty from the function $\mathbf{G}$ to the function $U\left(\mathbf{r}_{0}\right)$.

After some transformations, the problem under consideration, consisting of the equations of motion (1) and system (10) - (13), can be reduced to such working form:

$$
\begin{gathered}
(\mathbf{p} \cdot \dot{\boldsymbol{\xi}})-\left(\left\{\boldsymbol{\xi}-\mathbf{r}_{0}\right\} \cdot \nabla_{\mathbf{r}_{0}}\left(U\left(\mathbf{r}_{0}\right)\right)\right)=0 ; \\
\dot{\mathbf{r}_{0}}=\boldsymbol{\beta}(\mathbf{p}), \quad \dot{\mathbf{p}}=-\nabla_{\mathbf{r}_{0}}\left(U\left(\mathbf{r}_{0}\right)\right) ; \\
\left\{\boldsymbol{\xi}-\mathbf{r}_{0}\right\} \cdot\left\{\nabla_{\mathbf{r}_{0}}\left(U\left(\mathbf{r}_{0}\right)\right)+\boldsymbol{\Pi}\right\}=U\left(\mathbf{r}_{0}\right) ; \\
\mathbf{G}=-\nabla_{\mathbf{r}_{0}}\left(U\left(\mathbf{r}_{0}\right)\right), \\
\mathbf{F}=\widehat{\mu}\left(\boldsymbol{\Pi}+\nabla_{\mathbf{r}_{0}}\left(U\left(\tau, \mathbf{r}_{0}\right)\right)\right) .
\end{gathered}
$$

Equations (15) determine the dynamics of the point $\mathbf{r}_{0}$ of conditional localization of the excitation, if the function $U\left(\mathbf{r}_{0}\right)$ (dimensionless potential energy) is known. Equations (14), (16), (17), and (18) determine the vector function $\boldsymbol{\xi}$, scalar function $U\left(\mathbf{r}_{0}\right)$, as well as vectors $\mathbf{G}$ and $\mathbf{F}$ (dimensionless forces). Moreover, the relations (17), (18) for vectors $\mathbf{G}$ and $\mathbf{F}$, if the function $U\left(\mathbf{r}_{0}\right)$ is known, now are not the equations but equalities. That is, there are actually two equations: (14) and (16), defining functions $\boldsymbol{\xi}$ and $U\left(\mathbf{r}_{0}\right)$, as well as equations (15), which determine the dynamics of the quasiparticle.

\section{Results and Discussion}

\subsection{Statement of the problem for the potential field and analysis of its solution}

"Global" force $\mathbf{G}$ must have a physical nature, similar to a given external force $\boldsymbol{\Pi}$, in the sense that they both belong to the frame of reference of the crystal. That is why the force $\mathbf{G}$ must be a constant, and the energy $U\left(\mathbf{r}_{0}\right)$ can be defined by the equation: $U\left(\mathbf{r}_{0}\right)=-\left(\mathbf{G} \cdot \mathbf{r}_{0}\right)$. Now the constant $\mathbf{G}$ is to be determined, and the operation $\nabla_{\mathbf{r}_{0}}\left(U\left(\mathbf{r}_{0}\right)\right) \equiv-\mathbf{G}$ converts equality (17) into an identity. The remaining five of the relations given in (14) - (18) take the form:

$$
\begin{gathered}
(\mathbf{p} \cdot \dot{\boldsymbol{\xi}})+\left(\left\{\boldsymbol{\xi}-\mathbf{r}_{0}\right\} \cdot \mathbf{G}\right)=0 ; \\
\dot{\mathbf{r}_{0}}=\boldsymbol{\beta}(\mathbf{p}), \quad \dot{\mathbf{p}}=\mathbf{G} ; \\
(\boldsymbol{\xi} \cdot\{\boldsymbol{\Pi}-\mathbf{G}\})-\left(\mathbf{r}_{0} \cdot\{\boldsymbol{\Pi}-2 \mathbf{G}\}\right)=0 ;
\end{gathered}
$$




$$
\mathbf{F}=\widehat{\mu}(\mathbf{\Pi}-\mathbf{G}) .
$$

For constant force $\mathbf{G}$ there is only one non-trivial way of its selection: $\mathbf{G}=\frac{2}{3} \boldsymbol{\Pi}$. This selection is due to the fact that in equation (21) the constant factors: $\boldsymbol{\Pi}$ and $\mathbf{G}$, "are mixed"with multipliers: $\boldsymbol{\xi}, \mathbf{r}_{0}$, which depend on the time. If $\mathbf{G}=\frac{2}{3} \boldsymbol{\Pi}$, then the equation (21) takes the form, where they are separated. In this case (19) - (22) take the form:

$$
\begin{gathered}
(\mathbf{p} \cdot \dot{\boldsymbol{\xi}})+\frac{2}{3}\left(\left\{\boldsymbol{\xi}-\mathbf{r}_{0}\right\} \cdot \boldsymbol{\Pi}\right)=0 \\
\dot{\mathbf{r}_{0}}=\boldsymbol{\beta}(\mathbf{p}), \quad \dot{\mathbf{p}}=\frac{2}{3} \boldsymbol{\Pi} ; \\
\frac{1}{3}\left(\boldsymbol{\Pi} \cdot\left\{\boldsymbol{\xi}+\mathbf{r}_{0}\right\}\right)=\mathbf{0} \\
\mathbf{F}=\frac{1}{3} \widehat{\mu} \boldsymbol{\Pi} .
\end{gathered}
$$

Without loss of generality, the coordinate system can always be selected so that the external preset force was directed along the axis $x_{3}$ (axis $z$ ). Thus: $\Pi=(0,0, \Pi)$. Then the relations (23) and (25), from which we begin our analysis, take the form:

$$
\begin{gathered}
\frac{1}{3} \Pi\left(\xi_{3}+x_{3}^{0}\right)=0 ; \\
(\mathbf{p} \cdot \dot{\boldsymbol{\xi}})-\frac{2}{3} \Pi\left(x_{3}^{0}-\xi_{3}\right)=0 .
\end{gathered}
$$

Here $x_{3}^{0}$ is $z$-component of the vector $\mathbf{r}_{0}=\left\{x_{1}^{0}, x_{2}^{0}, x_{3}^{0}\right\}$, and $\xi_{3}$ is $z$-component of the vector $\boldsymbol{\xi}=\left\{\xi_{1}, \xi_{2}, \xi_{3}\right\}$. Taking into account that in the equations (27) $\Pi \neq 0$, the solution of the first (upper) of them can be written down with respect to $\xi_{3}: \xi_{3}=-x_{3}^{0}$. This solution is written relative to $\xi_{3}$, since the components $x_{\alpha}^{0} \quad(\alpha=1,2,3)$ of the vector $\mathbf{r}_{0}$ together with the momentum $\mathbf{p}$ are defined by equations (24). Therefore, prior of solve the second (lower) of the equations under consideration, which, taking into account the solution $\xi_{3}=-x_{3}^{0}$, takes the form:

$$
(\mathbf{p} \cdot \dot{\boldsymbol{\xi}})=\frac{4}{3} \Pi x_{3}^{0}
$$

it is necessary to analyze the solutions of the system (24).

To construct such a solution it is important to note that in the general case the motion of a quasiparticle in a constant field is always two-dimensional if it moves at an angle to the direction of the field. In this case, the motion is so that the direction vector of the field and the direction vector of motion always lie in the same plane, and the initial momentum of the quasiparticle (we denote it by q) has two components. In this case the direction of one of them coincides with the direction of the field. For example, it could be such a selection: $\mathbf{q}=\left\{\begin{array}{ll}0, & q_{2}, q_{3}\end{array}\right\}$. First, let us consider this case.

Thus, considering equations (24) and taking into account $\boldsymbol{\Pi}=\{0,0, \Pi\}$, lets write the second (right) of these equations in the components:

$$
\dot{p}_{1}=0, \quad \dot{p}_{2}=0, \quad \dot{p}_{3}=\frac{2}{3} \Pi .
$$

If $\mathbf{q}=\left\{0, q_{2}, q_{3}\right\}$, then the solutions of these equations are:

$$
p_{1}=0, \quad p_{2}=q_{2}, \quad p_{3}(\tau)=\frac{2}{3} \Pi \tau+q_{3} .
$$


I.e., the motion is really two-dimensional, because the condition $p_{1}=0$ is always fulfilled. The first (left) equation in (24), taking (29) into account, takes the form in components:

$$
\begin{gathered}
\dot{x_{1}^{0}}=\mathbf{0}, \quad \dot{x_{2}^{0}}=\sin \left(q_{2}\right), \\
\dot{x_{3}^{0}}=\sin \left(p_{3}(\tau)\right) .
\end{gathered}
$$

If at the moment of the field activation the quasiparticle was at the point $\mathbf{R}=\left\{\begin{array}{ll}R_{1}, R_{2}, R_{3}\end{array}\right\}$, then the general solution of equations (30) has the form:

$$
\begin{aligned}
& x_{1}^{0}=R_{1}, \quad x_{2}^{0}=R_{2}+\tau \sin \left(q_{2}\right), \\
& x_{3}^{0}=R_{3}+\frac{3}{2 \Pi}\left(\cos \left(q_{3}\right)-\cos \left(p_{3}(\tau)\right)\right) .
\end{aligned}
$$

Further, taking (29) into account, the equation (28) can be reduced to the form:

$$
q_{2} \cdot \dot{\xi}_{2}+\left(\frac{2}{3} \Pi \tau+q_{3}\right) \cdot \dot{\xi}_{3}=\frac{4}{3} \Pi x_{3}^{0} .
$$

And, given that $\xi_{3}=-x_{3}^{0}$, it reduces to a differential equation that determines the component $\xi_{2}$ :

$$
q_{2} \cdot \dot{\xi}_{2}=\frac{4}{3} \Pi x_{3}^{0}+\left(\frac{2}{3} \Pi \tau+q_{3}\right) \cdot \dot{x}_{3}^{0} .
$$

This equation can be integrated taking into account that component $x_{3}^{0}$ is fully defined in (31). But here, when considering a quasiparticle that moves at an angle to the direction of the field, it is sufficient to mark that the components $\xi_{2}$ and $\xi_{3}$ of the sought-for vector $\boldsymbol{\xi}$ are different from zero, and component $\xi_{1}$ is equal to zero. Then the amplitude part of the wave function corresponding to the quantum description of the situation in question $(\boldsymbol{\Pi}=\{0,0, \Pi\})$, takes the form $[8]$ :

$$
\varphi\left(\tau, x_{3}\right)=\sqrt[6]{\frac{4}{\left|F_{3}\right|}} A i\left(-\sqrt[3]{2\left|F_{3}\right|}\left\{x_{3}-2 x_{3}^{0}\right\}\right)
$$

where by the definitions ( 8$)$ and (26): $F_{3}=\mu_{3} \Pi / 3=\Pi / 3 \cos \left(p_{3}\right)$, and $p_{3}$ is defined in (29). It is obvious that this solution is invariant with respect to the orientation of the initial momentum of the form: $\mathbf{q}=\left\{q_{1}, 0, q_{3}\right\}$.

\subsection{Interpretation of anomalous dynamic splitting of the conduction electron energy in a strong electrostatic field}

Under the conditions of a real experiment, the injection of an electron into the conduction band can be carried out in such a way that the initial momentum of the electron is equal to zero: $\mathbf{q}=\left\{\begin{array}{lll}\mathbf{0}, & 0,0\end{array}\right\}$. This, for example, takes place for excitonic [10] or solitonic [11] injection methods, in particular, for polymers [10] and polymer-like [12] structures. In this case the solutions (29) take the form:

$$
p_{1}=0, \quad p_{2}=0, \quad p_{3}=\frac{2}{3} \Pi \tau,
$$

and solutions (31) are simplified to the form:

$$
\begin{gathered}
x_{1}^{0}=0, \quad x_{2}^{0}=0, \\
x_{3}^{0}=\frac{3}{2 \Pi}\left(1-\cos \left(\frac{2}{3} \Pi \tau\right)\right) .
\end{gathered}
$$

Here, without loss of generality, we can assume the following: $R_{1}=R_{2}=R_{3}=0$.

Then the system of equations (27) reduces to the form: 


$$
\frac{d}{d \tau}\left(\tau \xi_{3}\right)=x_{3}^{0}, \quad \xi_{3}=-x_{3}^{0}
$$

I.e. takes the form of two equations with respect to one unknown function $\xi_{3}$. In the classical case, such a redefinition is physically unacceptable. Therefore, one of the solutions would have to be deleted as unphysical. However, the function $\xi_{3}$ is an exclusively quantum parameter. Moreover, as noted above, it defines a "local" (in a reference system associated with a point $\mathbf{r}_{0}$ ) eigenvalue. In particular, here, in accordance with the definitions (7) and (26), this eigenvalue reduces to the form:

$$
\varepsilon=\xi_{3} \Pi / 3 .
$$

I.e. different functions $\xi_{3}$ correspond to different quantum states. For a detailed consideration of this problem, lets introduce two functions $\xi 0, \xi 1$ instead of the function $\xi_{3}$, each of which is determined by one of the equations (34). Namely:

$$
\frac{d}{d \tau}(\tau \cdot \xi 0)=x_{3}^{0} ; \quad \xi 1=-x_{3}^{0}
$$

Taking into account the explicit form of the function $x_{3}^{0}$, specified in (33), and integrating the first of the equations, one can obtain:

$$
\begin{aligned}
& \xi 0=\frac{3}{2 \Pi}\left(1-\frac{\sin \left(\frac{2}{3} \Pi \tau\right)}{\frac{2}{3} \Pi \tau}\right) \\
& \xi 1=\frac{3}{2 \Pi}\left(\cos \left(\frac{2}{3} \Pi \tau\right)-1\right) .
\end{aligned}
$$

For these two values, according to (35), we have two energies, the splitting between which is determined by the difference: $\Delta \varepsilon=\frac{\Pi}{3}(\xi 0-\xi 1)$, and has such an explicit form:

$$
\Delta \varepsilon=\frac{1}{2}\left(2-\frac{\sin (\theta)}{\theta}-\cos (\theta)\right) .
$$

Here we introduce the notation for generalized dimensionless time: $\theta \equiv \frac{2}{3} \Pi \tau$, which, according to (32), formally coincides with the dimensionless momentum $p_{3}$. Whereas $\tau \geq 0$, and the direction of the field $\Pi$ coincides with the positive direction of the axis $z$ (or $x_{3}$ ), thus $\theta>0$. In this case, each of the states $\varepsilon 0=\xi 0 \cdot \Pi / 3$ and $\varepsilon 1=\xi 1 \cdot \Pi / 3$ has "own"wave function:

$$
\begin{gathered}
\varphi 0\left(\tau, x_{3}\right)=\Lambda(\tau) A i\left(-\lambda(\tau)\left\{x_{3}-x_{3}^{0}+\xi 0\right\}\right), \\
\varphi 1\left(\tau, x_{3}\right)=\Lambda(\tau) A i\left(-\lambda(\tau)\left\{x_{3}-x_{3}^{0}+\xi 1\right\}\right),
\end{gathered}
$$

where $\Lambda(\tau) \equiv \sqrt[6]{4 /\left|F_{3}\right|}, \lambda(\tau) \equiv \sqrt[3]{2\left|F_{3}\right|}$. These notations emphasize that the field $F_{3}$, according to (8) and (26), is defined by: $F_{3}=\mu_{3} \Pi / 3=\Pi / 3 \cos \left(p_{3}\right)$, and, in accordance with (32), is a function of time. In addition, it follows from $(33),(36),(37)$ that the quantities $x_{3}^{0}, \xi 0, \xi 1$ are also depend on time.

Figure 1 shows the dependence (38) and its average value in the area of single-valuedness: $0 \leq \theta \leq \frac{\pi}{2}$ (for dynamic characteristics, such as speed, wave impulse, etc.).

The presence in Fig. 1 of splitting mean value (solid line) in addition to the dynamic splitting (dotted curve), is due to the duration of the process shown in Fig. 1 and is of the order of $t_{\max }=10^{-13} \mathrm{~s}$. At such short process times, the average splitting value is observed [10].

Let's show that $t_{\max }$ has the specified order of magnitude. Definition of dimensionless time $\tau$ was represented in [8]: $\tau=\frac{|M|}{\hbar} t$. For estimates, we can use the typical value of the width of the conduction band: $|M| \sim 1 \mathrm{eV}$. Taking into account that $\hbar=6.582 \cdot 10^{-16} \mathrm{eV} \cdot \mathrm{s}$, we can obtain an estimate for $\tau$ : 


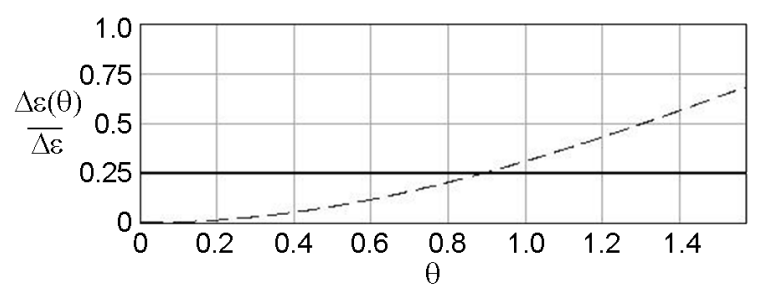

Fig. 1. Dynamics of anomalous splitting $\Delta \varepsilon(\theta)$ (dotted line) and its integral mean value $\overline{\Delta \varepsilon}$ (solid line) for an electron injected into the conduction band at zero initial momentum.

$$
\tau=\frac{t}{6.582 \cdot 10^{-16} \mathrm{~s}} .
$$

Maximum dimensionless time $\tau_{\max }$ is evaluated by the single-valuedness condition [1]: $\theta_{\max }=\frac{\pi}{2}$ or $\frac{2}{3} \Pi \tau_{\max }=\frac{\pi}{2}$. For the final evaluation $\tau_{\max }$ it is also necessary to estimate the dimensionless force $\Pi$. To do this, we also use the definition given in $[8]: \Pi=\frac{e D b}{|M|}$, which contains the following, except of $|M| \sim 1 \mathrm{eV}: e=1.6 \cdot 10^{-19} \mathrm{C}$ is electron charge; $b \sim 10^{-10} \mathrm{~m}=10^{-8} \mathrm{~cm}$ is crystal lattice constant; $D=925 \mathrm{kV} / \mathrm{cm} \sim 10^{6} \mathrm{~V} / \mathrm{cm}=10^{8} \mathrm{~V} / \mathrm{m}$ is applied field strength according to [10]. Then the estimate for $\Pi$ will be the following: $\Pi \sim 10^{-2}$. With this consideration we can obtain: $\tau_{\max }=\frac{3 \pi}{4 \Pi}=2,356 \cdot 10^{2}$. For a real dimensional time, according to (41), respectively, we can obtain: $t_{\max }=1.551 \cdot 10^{-13} \mathrm{~s}$.

A splitting analogous to the splitting, shown in Fig. 1, is discussed in [10]. There it is equal to $0.2 \mathrm{eV}$. In our consideration, all energies were normalized [8] to the width of the conduction band $|M| \sim 1 \mathrm{eV}$. Then, as follows from Fig. 1, the dimensional value of the mean splitting is equal to: $\overline{\Delta \varepsilon} \sim 0.25 \mathrm{eV}$. This qualitatively coincides with the splitting obtained in [10]. Moreover, if the considered dynamic splitting mechanism is single, then the width of the conduction band in the PNB polymer can be evaluated. Indeed, for the average value of the dimensional splitting obtained here completely coincides with the analogous splitting obtained in [10] for PNB polymers, the width of the conduction band should be $|M| \sim 0.8 \mathrm{eV}$.

There are other indications that the splitting observed in [10] has the dynamical nature under consideration. For example, in [10] it is mentioned that the presence of a field does not affect the occurrence of splitting. It follows from (38) that the field does not really affect the mean (observed) value of the splitting, regardless of the averaging method.

\subsection{Interpretation of anomalous dynamic "behaviour"of carrier}

Let us analyze certain features of the quantum description of an electron under the conditions of consideration (injection of an electron into the conduction band with an initial momentum equal to zero and a completely unified external field). Such an analysis is based on the wave functions (39), (40). They depend on time $\tau$ and "global" spatial variables associated with the frame of reference of the crystal: explicitly of the component $x_{3}$ and implicitly of the components $x_{1}, x_{2}$. In [8], quasi-stationary conditions were analyzed, for which the wave functions (39), (40) with a specified accuracy $\delta$ can be considered as stationary in some space-time domain. In some cases, it is possible to find such values $\delta$, under which the quasi-stationary conditions are satisfied at any time and at any point in space. Here is just such a case, because quasi-stationary conditions can be reduced to the form:

$$
\frac{d}{d \tau}\left|\varphi 0\left(\tau, x_{3}\right)\right|<\delta ; \quad \frac{d}{d \tau}\left|\varphi 1\left(\tau, x_{3}\right)\right|<\delta
$$

and integrated:

$$
\begin{aligned}
& \left|\varphi 0\left(\tau, x_{3}\right)\right|-\left|\varphi 0\left(0, x_{3}\right)\right|-\delta \tau<0 \\
& \left|\varphi 1\left(\tau, x_{3}\right)\right|-\left|\varphi 1\left(0, x_{3}\right)\right|-\delta \tau<0 .
\end{aligned}
$$



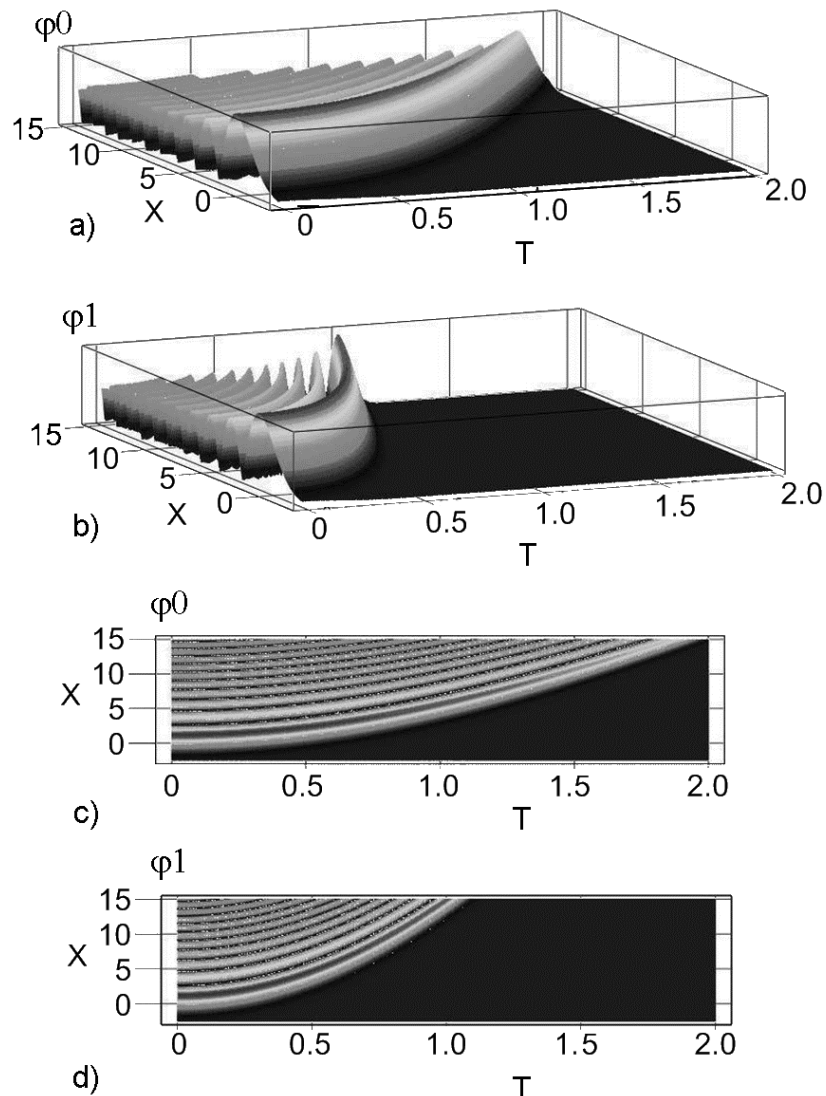

Fig. 2. Space-time distribution of wave functions $\phi 0(\mathrm{~T}, \mathrm{X})$ and $\phi 1(\mathrm{~T}, \mathrm{X})$. The following notations are introduced here: $\mathrm{T} \equiv \Pi \tau$ for a "time"variable, and $\mathrm{X} \equiv x_{3} \sqrt[3]{\Pi}$ for a "space"variable.

A numerical-graphical analysis of these relations shows that when $\delta \geq 3.3 \cdot 10^{-2}$ wave functions (39), (40) can be considered as stationary with the indicated accuracy for any $\tau$ and $x_{3}$.

A general form of the space-time distributions for wave functions (39), (40) is shown in Fig. 2. Plots 2(a), 2(b) show general forms of wave functions $\varphi 0, \varphi 1$ in a three-dimensional space. It can be seen that the electron density reduce "to the right" of the main maxima, i.e. practically absent. And the electron is actually localized in the area "to the left" of the main maxima.

However, these general forms are not very informative from the point of view of the analysis of physical results. Therefore, the plots $2(\mathrm{c}), 2(\mathrm{~d})$ are projections onto the plane $(\mathrm{T}, \mathrm{X})$ (the variables $\mathrm{T}, \mathrm{X}$ are defined in the caption to Fig. 2). It can be seen that the space-time configurations of the main maxima correspond with good accuracy to the classical trajectories of an electron motion in an external field. In addition, there are many more "non-main trajectories the probability of which is less than the main one.

It is also seen that if the main maxima are interpreted as trajectories, then in the state $\varphi 0$ mean electron velocity is half as much as in the state $\varphi 1$. That is, the effect of separation of electrons injected into different states should be observed. It can be observed if, for example, we include such a current suppression mechanism as the temperature. At a certain temperature and the free path of electrons from the injection point to the electrode, the contribution to the current from the "slow" component $\varphi 0$ must, under ideal conditions, suddenly disappear (in reality - to decrease rapidly). And the total current must decrease to a level determined only by the "fast"component.

It also follows from the plots 2(c), 2(d) that when the quasi-stationary conditions (42), (43) are fulfilled with the accuracy obtained $\left(\delta \geq 3.3 \cdot 10^{-2}\right)$, the electron is delocalized not only in space, which is already customary for quantum description, but also in time. This is due to the approximation of quasistationarity, which allows the amplitude part of the wave function to depend on time. I.e. in quantum analysis, an electron in its own frame of reference is delocalized not only in space, but also in time. 


\section{Conclusions}

In the article, obtained theoretical results were compared with the experimental data. It was shown that the previously developed method $[8,9]$ for agreed quantum and classical description of an electron in the conduction band explains the experiment [10] on the splitting of the conduction state in strong fields. Theoretical estimates show that, for a typical value of the width of the conduction band, $\sim 1 \mathrm{eV}$, the splitting is $\sim 0.25 \mathrm{eV}$, that is qualitatively coincides with the splitting obtained experimentally $(\sim 0.2 \mathrm{eV})$. On the other hand, it became possible to estimate the width of the conduction band for PNB polymers based on the splitting value. It consist $\sim 0.8 \mathrm{eV}$. The experimentally observed fact that the field magnitude does not affect the observed splitting value and arises immediately with the appearance of the field is confirmed. Analysis of the wave functions for the states obtained showed that in the region of the main maximum, the space-time distribution of the electron density, in the projection to the "coordinate-time" plane, reproduces the classical trajectory of the electron motion in the external field. Therewith there are a number of "non-main trajectories the probability of realization of which is less than the "main". It is shown that the electron is delocalized not only in space, but also in time. From the graphical-numerical realization of the theoretical results obtained, it follows that in one of the two states of the obtained splitting, the electron velocity is practically half that of the other. This can lead to anomalous current behavior when the temperature or other factors that suppress current are altered. For example, if the temperature increases, then for a certain its value, the mean free path of the "slow" component becomes less than the distance from the injection site to the electrode. In this case, the current under ideal conditions, suddenly (in reality very quickly) should decrease to a level determined only by the "fast" component.

\section{References}

1. A. D. Suprun, L. V. Shmeleva, Functional Materials, 19, 508 (2012).

2. A. Eremko, L. Brizhik, V. Loktev, Ann. of Phys., 369, 85 (2016).

3. A.Tur, G.Fruit, P.Louarn, V.Yanovsky, Phys. Plasmas, 21, 032113(16), (2014).

4. M. F. Smith, M. B. Walker, Phys. Rev. B, 67, 214509 (2003).

5. S. D. Sarma, S. Adam, E. H. Hwang et al., Rev. Mod. Phys., 83, 407 (2011).

6. O. P. Verkhoglyadova, B. T. Tsurutani, G. S. Lakhina, J. Geophys. Res.: Space Phys., 118, 7695 (2013).

7. A. V. Filippov, A. G. Zagorodny, A. I. Momot et al., Zh. Eksp. Teor. Fiz., 108, 497 (2009).

8. L. V. Shmeleva, A. D. Suprun, Functional Materials, 22, 524 (2015).

9. A. D. Suprun, L. V. Shmeleva, Functional Materials, 23, 443 (2016).

10. D. Huang, H. Li, J. Liu, Physica Status Solidi (b), 233, 197 (2002).

11. A. D. Suprun, L. V. Shmeleva, Functional Materials, 21, 69 (2014)

12. A. D. Suprun, L. V. Shmeleva, Nanoscale Res. Lett., 9, 200 (2014). 